\title{
Genomic and Epigenomic Profile of Uterine Smooth Muscle Tumors of Uncertain Malignant Potential (STUMPs) Revealed Similarities and Differences with Leiomyomas and Leiomyosarcomas
}

\author{
Donatella Conconi ${ }^{1, *,+}+\mathbb{D}$, Serena Redaelli ${ }^{1,+}+\mathbb{D}$, Andrea Alberto Lissoni ${ }^{1,2}$, Chiara Cilibrasi ${ }^{3}$, Patrizia Perego ${ }^{4}$, \\ Eugenio Gautiero ${ }^{5}$, Elena Sala ${ }^{5}$, Mariachiara Paderno ${ }^{1,2}$, Leda Dalprà ${ }^{1}$, Fabio Landoni ${ }^{1,2}$, \\ Marialuisa Lavitrano ${ }^{1}\left(\mathbb{D}\right.$, Gaia Roversi ${ }^{1,5}$ and Angela Bentivegna ${ }^{1, *} \mathbb{D}$
}

check for updates

Citation: Conconi, D.; Redaelli, S.; Lissoni, A.A.; Cilibrasi, C.; Perego, P.; Gautiero, E.; Sala, E.; Paderno, M.; Dalprà, L.; Landoni, F.; et al. Genomic and Epigenomic Profile of Uterine Smooth Muscle Tumors of Uncertain Malignant Potential (STUMPs)

Revealed Similarities and Differences with Leiomyomas and Leiomyosarcomas. Int. J. Mol. Sci. 2021, 22, 1580. https://doi.org/ $10.3390 /$ ijms 22041580

Academic Editors: Pasquale De Franciscis

Received: 21 December 2020

Accepted: 1 February 2021

Published: 4 February 2021

Publisher's Note: MDPI stays neutral with regard to jurisdictional claims in published maps and institutional affiliations.

Copyright: (c) 2021 by the authors. Licensee MDPI, Basel, Switzerland. This article is an open access article distributed under the terms and conditions of the Creative Commons Attribution (CC BY) license (https:// creativecommons.org/licenses/by/ $4.0 /)$.
1 School of Medicine and Surgery, University of Milano-Bicocca, 20900 Monza, Italy; serena.redaelli@unimib.it (S.R.); andreaalberto.lissoni@unimib.it (A.A.L.); m.paderno@campus.unimib.it (M.P.); leda.dalpra@unimib.it (L.D.); fabio.landoni@unimib.it (F.L.); marialuisa.lavitrano@unimib.it (M.L.); gaia.roversi@unimib.it (G.R.)

2 Clinic of Obstetrics and Gynecology, San Gerardo Hospital, 20900 Monza, Italy

3 Department of Biochemistry and Biomedicine, School of Life Sciences, University of Sussex, Falmer, Brighton BN1 9RH, UK; cc677@sussex.ac.uk

4 Division of Pathology, San Gerardo Hospital, 20900 Monza, Italy; patrizia.perego@asst-monza.it

5 Medical Genetics Laboratory, San Gerardo Hospital, 20900 Monza, Italy; e.gautiero@asst-monza.it (E.G.); elena.sala@asst-monza.it (E.S.)

* Correspondence: donatella.conconi@unimib.it (D.C.); angela.bentivegna@unimib.it (A.B.); Tel.: +39-0264488133 (A.B.)

+ Co-first authorship.

Abstract: Uterine smooth muscle tumors of uncertain malignant potential (STUMPs) represent a heterogeneous group of tumors that cannot be histologically diagnosed as unequivocally benign or malignant. For this reason, many authors are working to obtain a better definition of diagnostic and prognostic criteria. In this work, we analyzed the genomic and epigenomic profile of uterine smooth muscle tumors (USMTs) in order to find similarities and differences between STUMPs, leiomyosarcomas (LMSs) and leiomyomas (LMs), and possibly identify prognostic factors in this group of tumors. Array-CGH data on 23 USMTs demonstrated the presence of a more similar genomic profile between STUMPs and LMSs. Some genes, such as PRKDC and PUM2, with a potential prognostic value, were never previously associated with STUMP. The methylation data appears to be very promising, especially with regards to the divergent profile found in the sample that relapsed, characterized by an overall CGI hypomethylation. Finally, the Gene Ontology analysis highlighted some cancer genes that could play a pivotal role in the unexpected aggressive behavior that can be found in some of these tumors. These genes could prove to be prognostic markers in the future.

Keywords: uterine STUMP; array-CGH; copy number alterations; Genome Wide DNA Methylation

\section{Introduction}

According to the $2014 \mathrm{WHO}$, uterine smooth muscle tumors (USMTs) consist of a group of histologically heterogeneous and clinically diverse diseases ranging from malignant leiomyosarcoma (LMS) to benign leiomyoma (LM). In between the aforementioned, lies a heterogeneous group of rare mesenchymal tumors that cannot be clearly categorized as benign or malignant lesions. Uterine smooth muscle tumors of uncertain malignant potential (STUMPs) do not meet benign variant or true malignancy criteria, due to their variability in histologic appearance, immunohistochemical profile and clinical outcome [1]. 
The unusual combination of findings generally renders STUMP diagnosis very difficult, while the different criteria adopted by pathologists affect its accuracy [2].

Although the majority of patients with uterine STUMPs have good outcomes, unexpected aggressive behavior can be found in some of the tumors [2]. Because of the neoplasm's rarity, the etiology, prognostic factors, clinical outcomes and recurrence risks of these tumors are poorly defined [3]. Recently, a new classification of lesions according to genomic complexity has been described [4].

In this work, we analyzed the genomic profile of 23 USMTs by array-comparative genomic hybridization (array-CGH). In four samples the methylation profile was also investigated in order to find similarities and differences between STUMPs, LMSs and LMs, and possibly identify prognostic factors in this group of tumors.

\section{Results}

\subsection{Pathologic Features}

We performed an array-CGH analysis on 23 USMTs: 3 LMs, 14 STUMPs, 5 LMSs and 1 undifferentiated sarcoma (US), derived from a total of 20 patients who underwent first surgery between 1996 and 2013. The histological characteristics, the age at diagnosis and the type of surgery are listed in Table 1 (see also the Materials and Methods section). The median age at first diagnosis was 45.4 years old (ranging from 23 to 68).

Among the 14 STUMPs, 11/14 were primary tumors, of which four (STUMP1, STUMP3, STUMP4 and STUMP5) subsequently relapsed in either another STUMP or undifferentiated sarcoma (US) or LMS. For three patients it was possible to analyze both the primary STUMP and its recurrence (patients 4,5 and 6). Primary STUMPs of patients 8 and 9 and relapsed tumor of patient 7 were not available. Among the LMSs, three were primary (LMS2, LMS3 and LMS4), while two were relapsed tumors (LMS1 and LMS5). 
Table 1. Clinical features.

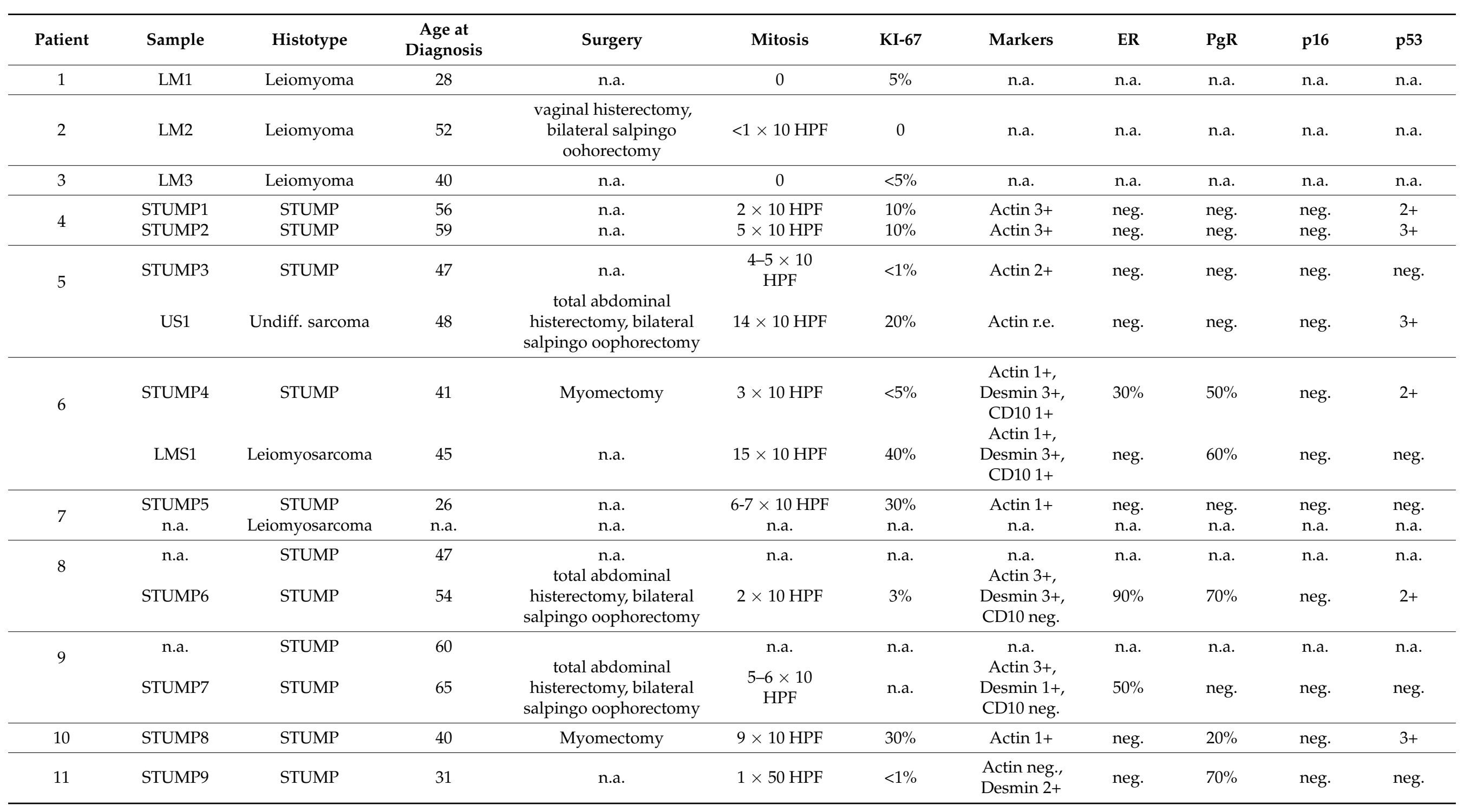


Table 1. Cont.

\begin{tabular}{|c|c|c|c|c|c|c|c|c|c|c|c|}
\hline Patient & Sample & Histotype & $\begin{array}{c}\text { Age at } \\
\text { Diagnosis }\end{array}$ & Surgery & Mitosis & KI-67 & Markers & ER & PgR & p16 & p53 \\
\hline 12 & STUMP10 & STUMP & 30 & Myomectomy & $\begin{array}{c}4-6 \times 10 \\
\mathrm{HPF}\end{array}$ & $<1 \%$ & Actin 2+ & $10 \%$ & $70 \%$ & neg. & neg. \\
\hline 13 & STUMP11 & STUMP & 42 & $\begin{array}{c}\text { total abdominal } \\
\text { histetectomy, left salpingo } \\
\text { oophorectomy }\end{array}$ & $\begin{array}{c}1-2 \times 10 \\
\mathrm{HPF}\end{array}$ & $<1 \%$ & Actin neg. & neg. & $40 \%$ & neg. & r.e. \\
\hline 14 & STUMP12 & STUMP & 47 & $\begin{array}{c}\text { total abdominal } \\
\text { histerectomy, bilateral } \\
\text { salpingo oophorectomy }\end{array}$ & $\begin{array}{c}4-5 \times 10 \\
\mathrm{HPF}\end{array}$ & $<5 \%$ & Actin 1+ & neg. & neg. & neg. & neg. \\
\hline 15 & STUMP13 & STUMP & 58 & $\begin{array}{c}\text { total abdominal } \\
\text { histerectomy, bilateral } \\
\text { salpingo oophorectomy }\end{array}$ & $\begin{array}{c}1-2 \times 10 \\
\mathrm{HPF}\end{array}$ & $<1 \%$ & Actin 1+ & neg. & $50 \%$ & neg. & neg. \\
\hline 16 & STUMP14 & STUMP & 34 & laparothomy & $\begin{array}{c}0-1 \times 10 \\
\mathrm{HPF}\end{array}$ & $1 \%$ & $\begin{array}{c}\text { Actin 3+, } \\
\text { Desmin 3+ }\end{array}$ & $30 \%$ & $80 \%$ & neg. & neg. \\
\hline 17 & LMS2 & Leiomyosarcoma & 49 & $\begin{array}{c}\text { total abdominal } \\
\text { histerectomy, bilateral } \\
\text { salpingo oophorectomy }\end{array}$ & $9 \times 10 \mathrm{HPF}$ & $30 \%$ & $\begin{array}{c}\text { Actin 2+, } \\
\text { Desmin 2+ }\end{array}$ & $20 \%$ & $90 \%$ & neg. & neg. \\
\hline 18 & LMS3 & Leiomyosarcoma & 63 & n.a. & $15 \times 10 \mathrm{HPF}$ & $10 \%$ & Actin 1+ & neg. & neg. & neg. & $3+$ \\
\hline \multirow[t]{2}{*}{19} & LMS4 & Leiomyosarcoma & 50 & $\begin{array}{l}\text { laparoscopic histerectomy, } \\
\text { bilateral adnexectomy }\end{array}$ & $\begin{array}{c}3-4 \times 10 \\
\text { HPF }\end{array}$ & $10 \%$ & $\begin{array}{l}\text { Actin 1+, } \\
\text { Desmin 1+ }\end{array}$ & neg. & $30 \%$ & neg. & r.e. \\
\hline & n.a. & Leiomyosarcoma & 54 & n.a. & n.a. & n.a. & n.a. & n.a. & n.a. & n.a. & n.a. \\
\hline \multirow{3}{*}{20} & n.a. & Leiomyosarcoma & 68 & n.a. & n.a. & n.a. & n.a. & n.a. & n.a. & n.a. & n.a. \\
\hline & LMS5 & Leiomyosarcoma & 73 & n.a. & $1 \times 10 \mathrm{HPF}$ & $<1 \%$ & Actin 1+ & $40 \%$ & $60 \%$ & r.e. & r.e. \\
\hline & n.a. & Leiomyosarcoma & 77 & n.a. & n.a. & n.a. & n.a. & n.a. & n.a. & n.a. & n.a. \\
\hline
\end{tabular}

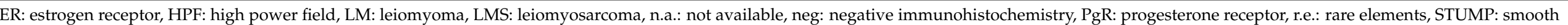
muscle tumors of uncertain malignant potential, US: undifferentiated sarcoma (more information in the Materials and Methods section). 


\subsection{Array-CGH Results}

STUMP samples revealed a wide range of copy number alterations (CNAs), from 17 to 365 , with a median value of 86.5 (Table 2).

Table 2. Array-CGH results.

\begin{tabular}{|c|c|c|c|c|c|c|}
\hline \multirow{2}{*}{ Patient } & \multirow{2}{*}{ Sample } & \multirow{2}{*}{ Follow Up } & \multirow{2}{*}{ OS } & \multicolumn{3}{|c|}{ CNAs } \\
\hline & & & & Total & Gain & Loss \\
\hline 1 & LM1 & NED & 6 & 110 & 106 & 4 \\
\hline 2 & LM2 & NED & 12 & 123 & 114 & 9 \\
\hline 3 & LM3 & n.a. & 2 & 268 & 239 & 29 \\
\hline 4 & $\begin{array}{l}\text { STUMP1 } \\
\text { STUMP2 }\end{array}$ & n.a. & 5 & $\begin{array}{l}25 \\
17 \\
\end{array}$ & $\begin{array}{l}23 \\
17 \\
\end{array}$ & $\begin{array}{l}2 \\
0 \\
\end{array}$ \\
\hline 5 & $\begin{array}{l}\text { STUMP3 } \\
\text { US1 }\end{array}$ & DOD & 2 & $\begin{array}{c}365 \\
28 \\
\end{array}$ & $\begin{array}{c}342 \\
28\end{array}$ & $\begin{array}{c}23 \\
0\end{array}$ \\
\hline 6 & $\begin{array}{l}\text { STUMP4 } \\
\text { LMS1 }\end{array}$ & DOD & 7 & $\begin{array}{l}256 \\
126\end{array}$ & $\begin{array}{l}238 \\
121\end{array}$ & $\begin{array}{c}18 \\
5\end{array}$ \\
\hline 7 & $\begin{array}{l}\text { STUMP5 } \\
\text { n.a. }\end{array}$ & DOD & 1 & 58 & 58 & 0 \\
\hline 8 & $\begin{array}{c}\text { n.a. } \\
\text { STUMP6 }\end{array}$ & ALIVE & 13 & 116 & 108 & 8 \\
\hline 9 & $\begin{array}{c}\text { n.a. } \\
\text { STUMP7 }\end{array}$ & NED & 8 & 94 & 88 & 6 \\
\hline 10 & STUMP8 & NED & 14 & 88 & 80 & 8 \\
\hline 11 & STUMP9 & ALIVE & 9 & 82 & 81 & 1 \\
\hline 12 & STUMP10 & n.a. & 10 & 40 & 38 & 2 \\
\hline 13 & STUMP11 & NED & 18 & 97 & 97 & 0 \\
\hline 14 & STUMP12 & NED & 10 & 156 & 155 & 1 \\
\hline 15 & STUMP13 & NED & 16 & 85 & 81 & 4 \\
\hline 16 & STUMP14 & NED & 7 & 59 & 58 & 1 \\
\hline 17 & LMS2 & ALIVE & 8 & 204 & 194 & 10 \\
\hline 18 & LMS3 & n.a. & n.a & 199 & 189 & 10 \\
\hline 19 & $\begin{array}{l}\text { LMS4 } \\
\text { n.a. }\end{array}$ & DOD & 7 & 262 & 234 & 28 \\
\hline 20 & $\begin{array}{l}\text { n.a. } \\
\text { LMS5 } \\
\text { n.a. }\end{array}$ & AWD & 23 & 166 & 148 & 18 \\
\hline
\end{tabular}

LM: leiomyoma, STUMP: smooth muscle tumors of uncertain malignant potential, LMS: leiomyosarcoma, US: undifferentiated sarcoma, NED: no evidence of disease, AWT: alive with tumor, DOD: dead of disease, OS: overall survival (years from the first tumor).

Two samples (STUMP3 and STUMP4) showed an exceptional number of CNAs (365 and 256 respectively). LMSs' total number of CNAs ranged from 126 to 262, with a median value of 199 . The only US sample analyzed showed the least number of CNAs compared to other sarcomas. Finally, LMs displayed an unexpected number of CNAs, ranging from 110 to 268 , with a median value of 123 . No statistically significant differences for the total number of CNAs were observed among the groups. A CNAs plot by chromosome for each patient is displayed in Figure S1.

Interestingly, combining these data with the follow-up status, we found that primary STUMPs and primary LMS derived from patients with a worst prognosis (dead of disease, 
DOD) displayed a higher number of CNAs compared to patients' samples with a good prognosis (alive with or without disease) (Figure 1A). As shown in Table 2, all patients with no evidence of disease (NED) had a longer disease.

ANOVA analysis performed on CNAs' distribution within chromosomes in LMs, STUMPs and LMSs revealed a statistically significant difference only in chromosome 17 (LMs vs. STUMPs, Tukey test $p=0.0004$; Figure 1B). This difference was also retained after splitting STUMPs in two different groups based on their ability to relapse (LMs vs. relapsing STUMPs, Tukey test $p=0.01$; LMs vs. not relapsing STUMPs, Tukey test $p=0.0008$, data not shown). No other differences were observed between the two groups of STUMPs or between STUMPs and LMSs.

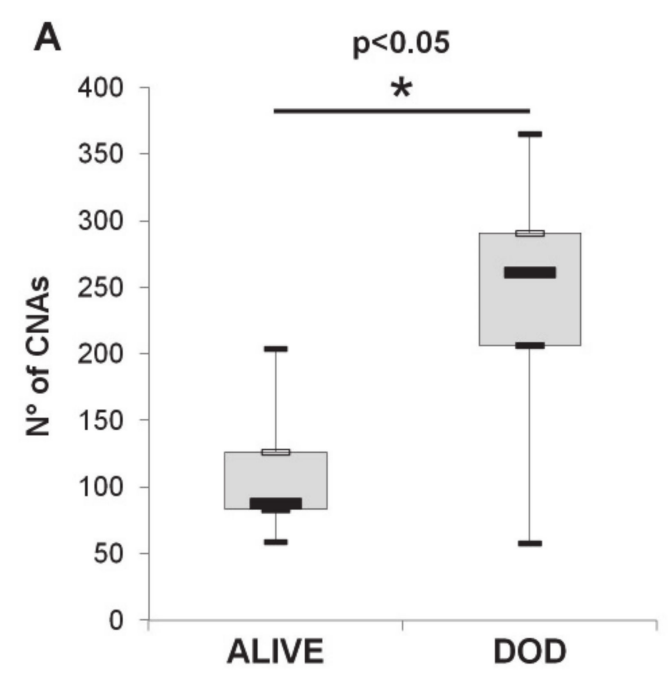

B

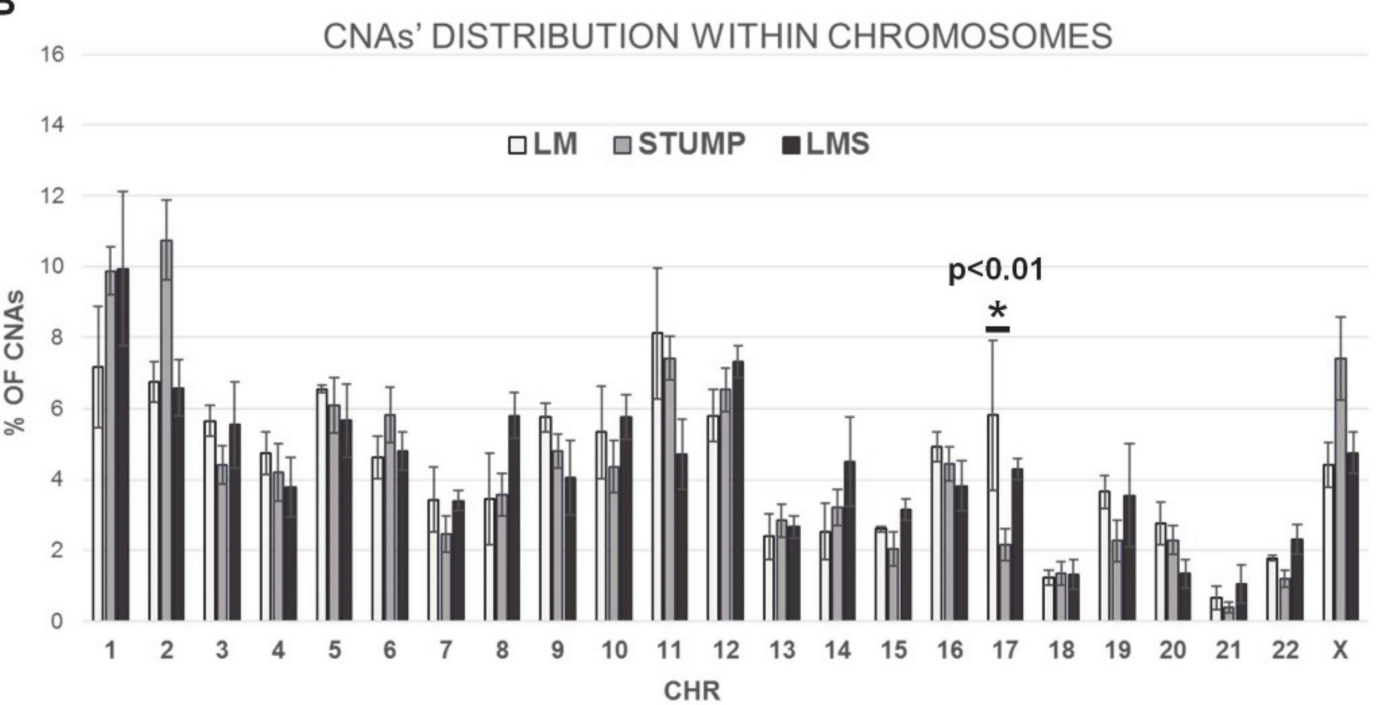

Figure 1. Array-CGH results. (A) Number of CNAs and follow-up status (Student $t$ test $p<0.05$ ). (B) CNAs' distribution within chromosomes in leiomyomas, STUMPs and leiomyosarcomas (LM vs. STUMPs, ANOVA and Tukey test $p=0.0004$ ). * statistically significant difference; - comparison between LM and STUMP

In order to decipher a possible common code between STUMPs and LMSs or between STUMPs and LMs, we further searched among the numerous CNAs detected for those shared among STUMPs and LMSs, or STUMPs and LMs (at least three samples, Table S1). CNAs common to all histotypes were excluded because they were not useful for setting the STUMP genomic profile (data not shown). For the same reason, CNAs shared only among STUMPs were included in the analysis. 
Curiously, losses represented a little percentage of the observed CNAs (6.2\%). The majority was exclusive; few were shared between two samples. Moreover, known cancerassociated genes were not found (data not shown).

Despite the high number of CNAs, only seven were shared by LMs and STUMPs. Conversely, STUMPs and LMSs shared 47 CNAs, in particular gain/amplification of the regions containing cancer related genes as PRKDC (8q11.21; eight samples), MLL3 (7q36.1; seven samples), ROCK2 (2p25.1; six samples), CTSB (8p23.1; six samples) and STAT2 (12q13.2; five samples) (Table S1 and Table 3). Interestingly, three out of four relapsing STUMPs shared an amplification of chromosome 2p24.1 including PUM2 gene, which was not identified in other samples.

Narrowing down the analysis to the relapsing STUMPs and LMSs, we found some shared CNAs, especially in STUMP4 (the only relapsed in a LMS), including gain/amplification of the regions encompassing STAG1 (3q22.2-22.3; four samples), RFC4 (3q27.3; four samples), CCRN4L and ELF2 (4q31.1; three samples) (Table 3).

As the amplification of the BCL2 gene in primitive STUMPs and their corresponding relapsed tumors have been previously reported [5], an analysis of BCL2 data was performed, revealing copy number gain or amplification in 13 samples: one leiomyoma (LM2), four primary STUMPs (STUMP10, STUMP11, STUMP12, STUMP13), two relapsing STUMPs (STUMP3 and STUMP4), one relapsed STUMP (STUMP6), three primary leiomyosarcomas (LMS2, LMS3 and LMS4) and two relapsed LMSs (LMS1 and LMS5) (Table S1 and Table 3).

Table 3. Top nine CNAs shared between STUMPs and leiomyosarcomas (all copy number gains).

\begin{tabular}{|c|c|c|c|c|c|c|c|c|c|c|}
\hline & $P R K D C$ & $M L L 3$ & ROCK2 & CTSB & STAT2 & STAG1 & RFC4 & $\begin{array}{c}\text { CCRN4L, } \\
\text { ELF2 }\end{array}$ & PUM2 & BCL2 \\
\hline STUMP2 & + & & & & & & & & & \\
\hline STUMP3 & + & + & + & + & & & & & + & + \\
\hline STUMP4 & & + & & & + & + & + & + & + & + \\
\hline STUMP5 & & & & & & & & & + & \\
\hline STUMP6 & & & & + & & & & & & + \\
\hline STUMP7 & + & + & & + & & & & & & \\
\hline STUMP9 & & & + & & & & & & & \\
\hline STUMP10 & & & & & & & & & & + \\
\hline STUMP11 & & & + & + & & & & & & + \\
\hline STUMP12 & + & & + & & & & & & & + \\
\hline STUMP13 & & & + & & & & & & & + \\
\hline LMS1 & + & + & & & + & & & & & + \\
\hline LMS2 & + & & & + & & + & + & & & + \\
\hline LMS3 & + & + & + & & + & & + & + & & + \\
\hline LMS4 & & + & & & + & + & + & & & + \\
\hline LMS5 & + & + & & + & + & + & & + & & + \\
\hline
\end{tabular}

\subsection{Promoter $C p G$ Island Methylation}

We evaluated the CpG island methylation status of four samples, two STUMPs (STUMP4, STUMP8) and two LMSs (LMS1 and LMS2). STUMP8 and LMS2 were primary lesions that had not yet relapsed, while LMS1 was the relapse of STUMP4. The array platform used in this study covers 27,800 CpG Island (CGIs) of the human genome and all the data (percentages and frequencies) are referred to the total number of CGIs included in the array. We calculated the percentages of unmethylated/methylated/undetermined CGIs and we observed that STUMP4, the primary lesion that relapsed, had a divergent methylation pattern compared to the other three samples (Figure 2A, $p<0.01$ Student's $t$ test). In particular, the percentage of methylated CGIs for all chromosomes in STUMP4 was significantly lower $(p<0.01$, Chi square test) compared to the one observed in the 
other three samples, which presented similar percentages, around 50\%, of methylated and unmethylated CGIs (Figure 2B).

A

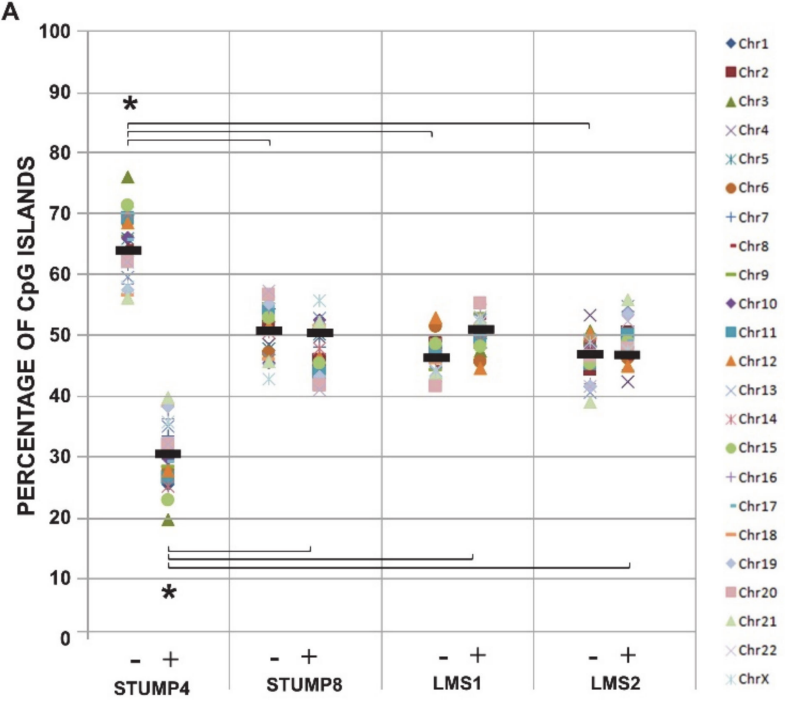

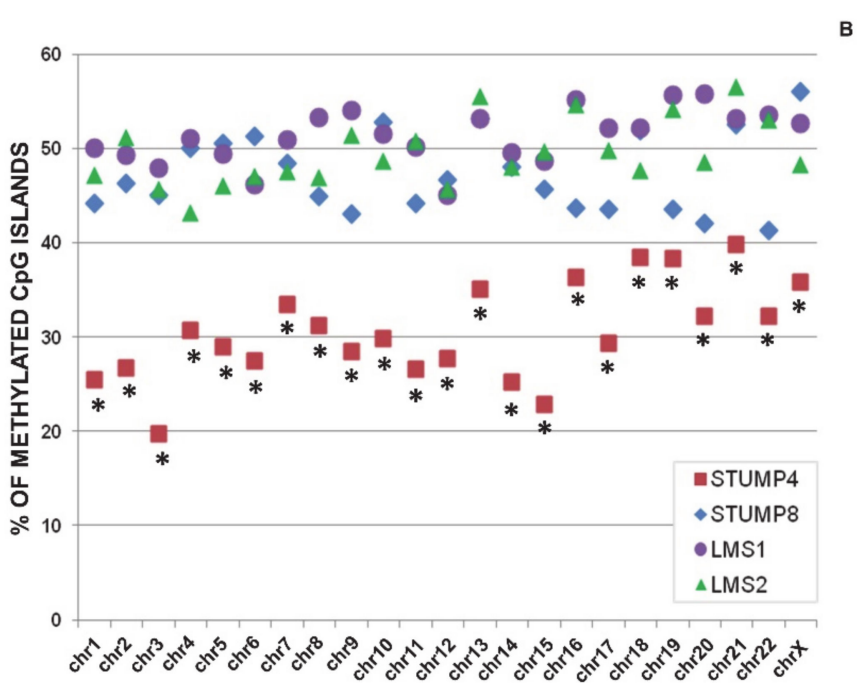

Figure 2. Methylation results. (A) Frequency of unmethylated ( - ) and methylated (+) CGIs for each sample. The methylation status for each chromosome is reported. Mean values are indicated with black dashes. STUMP4 vs. other samples ${ }^{*} p<0.01$, Student's $t$ test. (B) Frequency of methylated CGI islands for each chromosome in each sample. STUMP4 vs. other samples ${ }^{*} p<0.01$, Chi-square test.

In order to perform a Gene Ontology (GO) analysis, we set up two lists of genes on the basis of the promoter methylation status. The first list included the 667 genes which were methylated, while the second one the 2220 genes that resulted unmethylated in all samples. Cancer-related GO terms were grouped in different functional categories, as previously described [6]. Each category was scored based on its own percentage of genes belonging to that specific category and normalized to the total number of genes. 'Metabolism' and 'transcription and gene expression' were the most represented categories in both lists of methylated and unmethylated genes. However, no statistically significant differences were identified (data not shown).

We subsequently refined our analysis, limiting the GOgroups to 'biological process' aspects (see Materials and Methods section). The most significant GO-groups included 336 genes $\left(p\right.$-value $\left.=1.92 \times 10^{-35}\right)$ and 99 genes $\left(p\right.$-value $\left.=1.68 \times 10^{-9}\right)$ from the unmethylated and methylated gene lists respectively. We found that gene associated GOterms such as 'transcription and gene expression', 'cell cycle' and 'cell signalling' were detected in both the unmethylated and methylated genes' lists. Conversely, GOterms such as 'transport' and 'DNA repair and chromatin remodeling' and 'development and morphogenesis' and 'cell death and apoptosis' were found almost exclusively in the unmethylated or methylated group of genes, respectively.

Given the divergent distribution of CGI methylation percentages in STUMP4, we then compared its methylation pattern to the one of the other three samples. We found that 943 unmethylated genes and 180 methylated genes were exclusive to STUMP4, suggesting a specific methylation signature for this tumor. We restricted the analysis to 'biological process' aspects observing that 236 of the unmethylated genes in the most significant GOgroup $\left(p\right.$-value $=2.33 \times 10^{-11}$ ) were mainly involved in 'transcription and gene expression', 'metabolism', 'cell signaling', 'cell cycle' and 'cell death and apoptosis' categories, as verified in the previous analysis. Surprisingly, only four of the methylated genes were included in the most significant GO-group ( $p$-value $=0.00648$ ) and were involved in 'cytoskeleton organization'. Furthermore, statistical analysis (chi-square test) revealed a significant difference for some categories between STUMP4 and the other samples (Figure 3). 


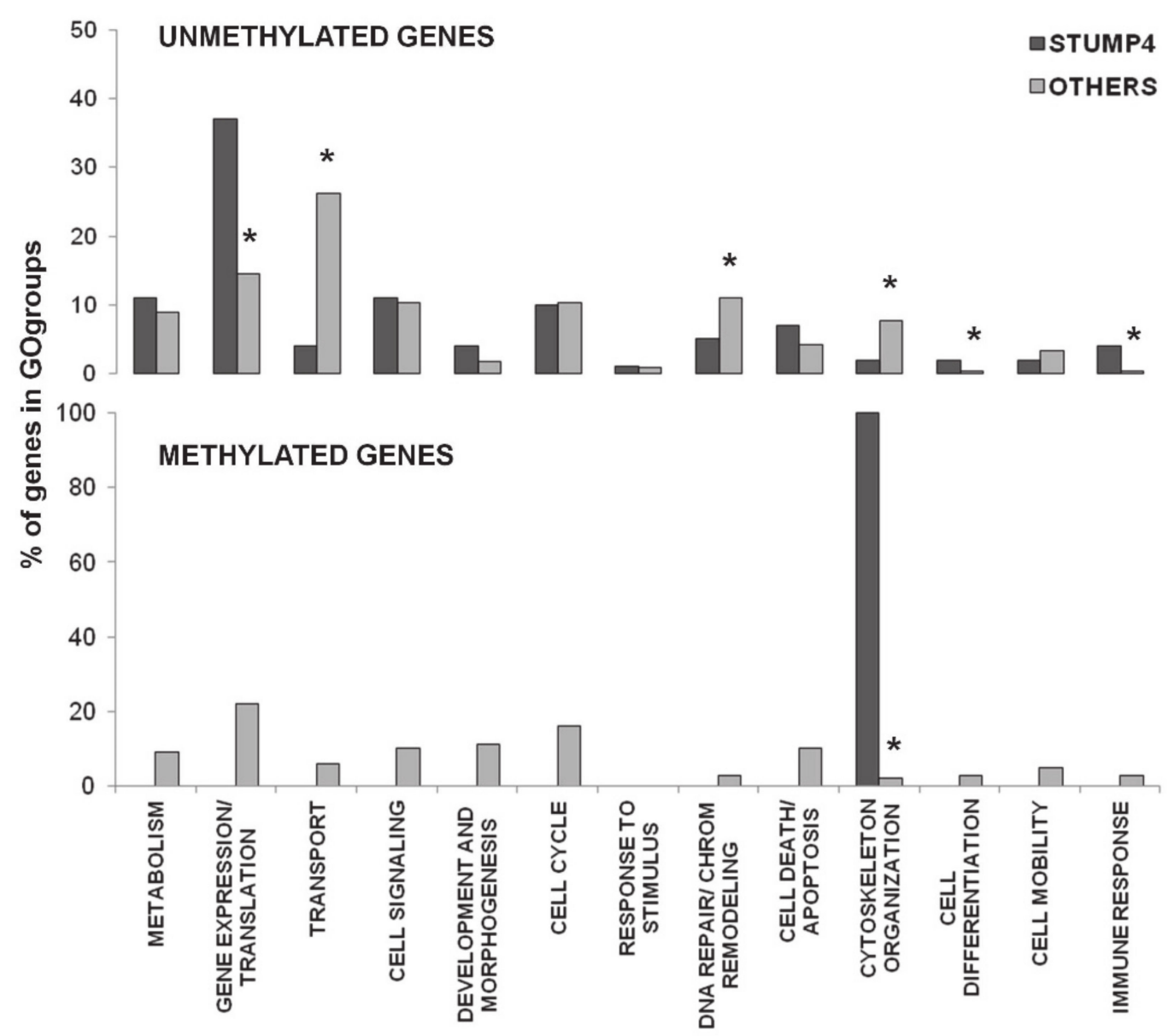

Figure 3. Gene Ontology results. Percentage of unmethylated and methylated genes in GOgroups. ${ }^{*} p<0.05$.

The analysis" results allowed to speculate on the potential activated "cell signalling" pathways among the samples. For example, IK beta kinase/NFK beta cascade was mainly represented in STUMP4's exclusively unmethylated gene list, while the Wnt signalling pathway was detected in the list of genes found to be methylated in all the samples (Table S2).

Afterwards, we focused on the different methylation patterns between the STUMP primary lesion and its recurrence (STUMP4 vs. LMS1). In order to perform the Gene Ontology analysis, we established two lists of genes. The first list included 628 genes, which were found methylated in STUMP4 and unmethylated in LMS1. Conversely, in the second list 2972 genes were reported resultingunmethylated in STUMP4 and methylated in LMS1. As expected, 'metabolism' and 'transcription and gene expression' were the most represented biological functions in both samples, but no statistically significant differences were identified (data not shown). However, the percentage of unmethylated promoter CGIs in the 'Cell signalling' category differed in the two samples: STUMP4 displayed $3.1 \%$ of unmethylated genes (92/2972) compared to $9.2 \%(58 / 628)$ of LMS1 $(p<0.01$, chi square test). In conclusion, we may assume that Ras protein signal transduction, Wnt signalling pathway and NFKbeta cascade were more involved in the primary lesion than in its respective recurrence, being more represented in STUMP4 unmethylated promoters (Table 4). 
Table 4. Cell signaling Pathways with the related genes in STUMP4 and its recurrence LMS1.

\begin{tabular}{|c|c|c|c|}
\hline Cell Signaling Pathway & $\begin{array}{c}\text { Genes with a Significant } \\
\text { GOterm }\end{array}$ & $\begin{array}{l}\text { Unmethylated Genes in STUMP4 } \\
\text { (Methylated in LMS1) }\end{array}$ & $\begin{array}{c}\text { Methylated Genes in } \\
\text { STUMP4 (Unmethylated in } \\
\text { LMS1) }\end{array}$ \\
\hline NFK $\beta$ cascade & 22 & $\begin{array}{l}\text { NDFIP1, MAP3K7IP2, PLK2, NDFIP2, } \\
\text { NLRC3, PPM1A, SECTM1, TRIM13, } \\
\text { IRAK1, TSPAN6, VAPA, TNFAIP3, } \\
\text { BIRK2, C9orf89, EDG2, GOLT1B, } \\
\text { LTBR, PRDX3, TNFRSF1A, RPS6KB2 }\end{array}$ & ZDHHC13, TFG \\
\hline $\begin{array}{l}\text { WNT receptor signaling } \\
\text { pathway }\end{array}$ & 16 & $\begin{array}{c}\text { CSNK2B, LRRFIP2, PYGO2, PORCN, } \\
\text { DKK1, FZD3, FZD6, FZD8, HBP1, } \\
\text { WNT10A, WNT2B, WNT6, WNT7A, } \\
\text { DIXDC1, TLE4, WNT11 }\end{array}$ & / \\
\hline $\begin{array}{l}\text { Smoothened signaling } \\
\text { pathway (Hedgehog } \\
\text { signaling pathway) }\end{array}$ & 1 & CTNNA1 & / \\
\hline $\begin{array}{l}\text { Negative regulation of } \\
\text { WNT pathway }\end{array}$ & 1 & $F R Z B$ & / \\
\hline $\begin{array}{l}\text { Regulation ARF GTPase } \\
\text { activity (Ras protein } \\
\text { transduction) }\end{array}$ & 24 & $\begin{array}{c}\text { ARGEF2, PSD3, CENTB2, CENTD1, } \\
\text { GNB1, DOK3, EVI5, PSD4, RASSF1, } \\
\text { RASSF6, GBF1, DDEF1, DOK2, } \\
\text { SMAP1, PSCD1, PSCD4, ARFGEF1, } \\
\text { CTGLF4 }\end{array}$ & $\begin{array}{c}\text { ARFGAP1, FBXO8, IQSEC1, } \\
\text { TBC1D22A, TBC1D15, } \\
\text { TBC1D16 }\end{array}$ \\
\hline Ras GTPase activity & 15 & $\begin{array}{c}\text { TBC1D5, TBC1D8, TBC1D9, } \\
\text { TBC1D9B, TBC1D2B, TBC1D4, EVI5L, } \\
\text { SGSM1, TBC1D10A, TBC1D20, } \\
\text { TBC1D25, TBC1D10C, TBC1D12, } \\
\text { CENTG1, RABGAP1 }\end{array}$ & / \\
\hline
\end{tabular}

\section{Discussion}

The World Health Organization classifications indicate that uterine smooth muscle tumors that cannot be histologically diagnosed as unequivocally benign or malignant should be termed STUMP [7]. For this reason, many authors are working to obtain a better definition of diagnostic and prognostic criteria. Several immunohistochemical markers have been analyzed, as well as genetic alterations, but a definitive response has not been obtained yet.

We reported an array-CGH analysis performed on 23 USMTs, including 14 STUMPs, in order to extend the genomic knowledge on this type of tumor. A similar analysis, on a comparable number of STUMPs, has been previously reported [4] identifying a genomic index, based on array-CGH data, as a recurrence predictor. However, we did not find that the genomic index is a recurrence predictor for our patients.

We found that the number of CNAs correlates with primary STUMPs and LMSs prognosis. In fact, samples derived from patients with a worse prognosis displayed a higher number of CNAs compared to the ones derived from patients with a good one.

In order to identify CNAs with a potential prognostic value, we selected the most frequently shared CNAs between STUMP and LMS samples. Array-CGH data showed a high number of gains and a very low number of losses in these lesions. Our hypothesis is that genomic losses would be hidden due to a previous step of genome endoreduplication, as we previously suggested [5]. The presence of polysomy for chromosomes 3, 7 and 17 detected by fluorescence in situ hybridization support this hypothesis (Figure S2).

It is already known that copy number gains or amplifications are associated with protein overexpression in cancer. Eight samples (four STUMPs and four LMSs) shared gain or amplification of PRKDC (DNA-Dependent Protein Kinase Catalytic Subunit). This gene 
is a key component of the non-homologous end joining pathway for DNA repair and its overexpression and/or copy number gain have been observed in several cancer types, such as lung cancer, liver cancer and colorectal cancer, and are associated with more advanced tumor grade and poor survival [8,9]. Recently, its high expression has been associated with poor survival in gastric cancer patients [10] and in both treated and untreated breast cancer patients [11]. In our samples, copy number gain was not associated with poor prognosis; however, it was not identified in leiomyomas. This makes it a very interesting target.

MLL3 (Mixed Lineage Leukemia 3) copy number gain or amplification was shared among seven samples (three STUMPs and four LMSs). Intriguingly, two STUMPs subsequently relapsed and the third was a relapsed STUMP. Mutations of this gene were reported in different tumors [12], but not in STUMP or leiomyosarcoma.

ROCK2 and CTSB gains or amplifications were shared among six samples (both STUMPs and LMSs). ROCK2 (Rho associated coiled-coil containing protein kinase 2) upregulation has been described in human glioblastoma CSCs [13], in gastric cancer [14], as well as in ovarian cancer samples [15]. Moreover, ROCK2 expression level has been associated with worse prognosis in osteosarcoma tissues [16]. CTSB (cathepsin-B) overexpression has been identified in several tumors with different effects on patient survival [17]. However, high expression of CTSB has been related to poor survival in glioblastoma patients and involved in promoting temozolomide intrinsic resistance [18].

STAT2 has a well-known role in the anti-viral, immunomodulatory, anti-apoptotic and anti-proliferative effects of IFN-I [19]. Moreover, its high expression in different tumors, such as melanoma, colon adenocarcinoma, breast cancer and lung cancer, has been associated with poor prognosis, highlighting it as a promising therapeutic target [20]. In our samples, its gain/amplification was identified in one relapsing STUMP and its recurrence and in three LMSs, both primary and relapsed.

Interestingly, three out of four relapsing STUMPs shared an amplification of the PUM2 gene. PUM2 is an RNA-binding protein involved in embryonic development, cell differentiation and stem cell proliferation. Its role has also been reported in several tumors, such as glioblastoma, where it was overexpressed and promoted cell proliferation [21], or breast tumor, where it promoted the stemness of cancer cells [22].

We refined the analysis comparing only the relapsing STUMPs with LMSs, and we found some shared CNAs. In particular, we observed a copy number gain in four samples (one STUMP and three LMSs) involving STAG1 (Stromal Antigen 1). Its amplification and overexpression in breast and ovarian cancer cell lines has been previously demonstrated [23].

The same STUMP sample (the only relapsed in a LMS) shared RFC4 (Replication Factor C Subunit 4) gain with three LMSs. Overexpression of RFC4 commonly occurs in colorectal cancer and higher levels of RFC4 protein expression correlate with a worse overall survival [24]. Moreover, its overexpression in tumor tissues predicted poor survival in hepatocellular carcinoma and it was also considered a potential therapeutic target [25].

Finally, ELF2 (E74 Like ETS Transcription Factor 2) copy number gain was shared among only three samples, but its role in tumorigenesis has been well described. In fact, overexpression of ELF2 enhanced tumor cell proliferation [26] in nasopharyngeal carcinoma, while its silencing had the opposite effect [27].

We previously identified the amplification of BCL2 gene in primitive STUMPs and their corresponding relapsed tumors [5]. This observation has not been confirmed in this enlarged cohort, where BCL2 amplification was observed in different samples (eight STUMPs and five LMSs), not only the ones that relapsed. It is therefore clear that an increase of analyzed cases is crucial for the validation of new targets. In this context, we collected and examined 14 STUMPs, a significant number for this rare type of tumor.

Epigenomic modifications, such as DNA methylation, are an integral part of the molecular determinants, contributing to malignancy [28]. For the first time in our knowledge, we mapped out a methylation profile for this type of tumors. Considering the global 
DNA methylation data, an overall CGI hypomethylation of the STUMP that relapsed was noticed compared to the other three samples. Although the analysis was performed on few cases (two STUMPs and two LMSs), it is interesting that this finding is similar to the one we previously published on three glioma stem cell lines [6]. This analogy could be explained by a greater presence of cancer stem cells in STUMP4, which would justify the worse prognosis with relapse and death of the patient.

In cancer, the role of promoter hypermethylation in silencing tumor suppressor genes and its impact on tumor initiation, progression and prognosis are well known. Even though DNA hypomethylation was the first epigenetic disruption in cancer [29], it took more studies to define its implications in tumorigenesis leading to genomic instability [30], enhanced expression [31], loss of imprinting [32] and abnormal X-chromosome activation [33]. It is interesting to note that the total number of CNAs of STUMP4 is doubled compared to its recurrence and threefold compared to the other STUMP, confirming the association between DNA hypomethylation and genomic instability.

Our data showed a threefold of genes with unmethylated promoter CGIs compared to the methylated ones, considering all four samples. As expected, no statistically significant differences were highlighted by the Gene Ontology analysis comparing the four samples. Conversely, significant differences for several categories emerged from the isolation of the sample with a different profile of methylation (STUMP4), and the comparison with the other three. This analysis highlighted specific signaling pathways that might be preferentially activated in this tumor. The same conclusion emerged thanks to the comparison of the primary sample with its recurrence, outlining a distinctive behavior of the sample with the worst prognosis.

In conclusion, our data demonstrated a more similar genomic profile between STUMPs and LMSs with some genes with a potential prognostic value. Despite the lack of an NGSbased point mutations investigation, which would have given a complete picture of the mutation burden (the landscape), and the number of samples being too small to draw conclusions, the Gene Ontology analysis highlighted some cancer genes that could play a pivotal role in the unexpected aggressive behavior in some of these tumors. These genes could prove to be prognostic markers in the future. Finally, the methylation data appears to be very promising, especially with regards to the divergent profile found in the sample that relapsed, despite the analysis being restricted to only four samples.

\section{Materials and Methods}

\subsection{Tumor Samples/Patients}

A total of 23 uterine smooth muscle tumors: 3 leiomyomas, 14 STUMPs, 5 leiomyosarcomas and 1 undifferentiated sarcoma, were collected from a total of 20 patients (Table 1). Comitato Etico della provincia Monza e Brianza approval and written informed consent was obtained. Patients were selected by a team of pathologists with high level of expertise in the field of soft tissues gynecological neoplasms, supported by a team of gynecologic oncologists with high level of expertise in soft tissue sarcoma.

In our case, we based the diagnosis of STUMP following the WHO indication: a uterine SMT that cannot be diagnosed unequivocally as malignant (at least two of the following criteria: diffuse moderate-to-severe atypia, a mitotic count of at least 10 mitotic figures /10 HPF, tumor cell necrosis, as defined by Bell et al. [34]) or clearly benign.

As far as we are concerned, all the specimens defined as STUMP had $<10$ mitosis/10 HPFs, but were not classified as benign because of the presence of tumor necrosis even without atypia or additional histological parameters that may predict adverse outcome, as reported by Gupta et al. [35] (briefly: remarkable atypia and borderline mitosis, atypical mitoses, epithelioid differentiation, vascular involvement, infiltrative/irregular margins).

\subsection{Immunohistochemistry}

Immunohistochemical staining was performed on FFPE ( $4 \%$ formalin) sections of $1-\mu \mathrm{m}$ thickness. The entire pre-treatment process of deparaffinization, rehydration and 
epitope retrieval was performed using PT LINK (Dako, supplied by Agilent Technologies, Santa Clara, CA, USA). Then the sections were placed into the Autostainer Link 48 with the EnVision FLEX visualization system.

Estrogen receptor (ER), progesterone receptor (PgR), p16, p53, Ki67 (for mitotic activity) and other markers were evaluated (all antibodies were purchased from Dako, except for p16 antibody that was from Gennova Scientific, Sevilla, Spain). For interpretation of immunohistochemical staining, the system proposed by Ip et al. [36] was adopted. In particular, the percentage of positive nuclei for ER, PgR and Ki67 and a scale of positivity (neg: no staining, $1+:<33 \%$ of positive cells, $2+: 33-66 \%$ of positive cells, $3+:>66 \%$ of positive cells) for p16, p53, myogenic markers (actin, desmin) and CD10 were reported in Table 1. For p16, either strong nuclear or cytoplasmic staining or a combination, was considered positive. For p53, only nuclear staining was considered positive.

\subsection{DNA Extraction from FFPE Tissues}

DNA extraction from Formalin-Fixed Paraffin-Embedded (FFPE) tissues was performed by Invisorb ${ }^{\circledR}$ spin Tissue Mini Kit (Stratec Molecular GmbH, D-13125, Berlin, Germany) using $10 \mu \mathrm{m}$ slices from formaline-fixed paraffin embedded biopsies. All DNAs were quantified by Nanodrop-1000 (ThermoFisher, Wien, Austria) and DNA integrity was measured through agarose gel electrophoresis; generally all FFPE DNA appeared partially fragmented.

\subsection{Array Comparative Genomic Hybridization (Array-CGH)}

Sample preparation, slide hybridization and analysis were performed using SurePrint G3 Human CGH Microarray $8 \times 60 \mathrm{~K}$ (Agilent Technologies, Santa Clara, CA, USA) based on the UCSC Genome Browser hg18, NCBI build 36.1, March 2006 with 41Kb overall median probe spacing ( $33 \mathrm{~Kb}$ in RefSeq genes), according to the manufacturer's instructions for FFPE samples. A control female DNA from a healthy female blood donor was used as sex-match reference. This control DNA was validated by testing on CGH microarray using as references available commercial European Male DNA NA-1289 and European Female DNA NA-12878 (Coriell Institute, Camden, NJ, USA) and by self-hybridization. All the samples were heat fragmented at $99^{\circ} \mathrm{C}$ for 10 to $20 \mathrm{~min}$ and $250 \mathrm{ng}$ of fragmented DNA per sample were directly labelled by Genomic DNA ULS-labelling Kit (Agilent Technologies) according to the protocol. ULS-labelling kit does not copy or amplify the input DNA, so the yield after the labelling is the same as the input DNA. The arrays were scanned at $2 \mu \mathrm{m}$ resolution using the Agilent microarray scanner and analyzed using Feature Extraction v10.7 and Agilent Genomic Workbench v5.0 softwares (Agilent Technologies, Santa Clara, CA, USA). The Aberration Detection Method 2 (ADM-2) algorithm was used to compute and assist the identification of aberrations in a given sample (threshold $=5.0)$, assigning a statistical score based on the average quality weighted log ratio (DLRS) of the sample and reference channels. ADM2 threshold filter selected at least three contiguous probes with an average absolute value of the log2ratio change of 0.60 across the aberration in order to define gains and losses. Additional abnormalities were manually noted when detected via visual inspection of the experimental data points for each chromosome. The estimated percentage of mosaicism was calculated using the formula determined by Cheung SW et al. [37].

\subsection{MeDIP-Chip}

Methylated DNA immunoprecipitation and chip hybridization were performed following the guidelines of Agilent Microarray Analysis of Methylated DNA Immunoprecipitation Protocol (Version 1.0, Agilent Technologies, Santa Clara, CA, USA) as previously described [6]. Briefly, purified genomic DNA was sonicated to obtain fragments of 200-600 bp in size and $5 \mu \mathrm{g}$ of sheared DNA was immunoprecipitated using $50 \mu \mathrm{l}$ of pan-mouse IgG Dynal magnetic beads (Life Technologies Italia, Monza, Italy) and $5 \mu \mathrm{g}$ of 5-methylcytosine antibody (Eurogenetec, Seraing, Belgium). MeDIPed DNA and reference DNA were purified and directly labelled with Cyanine 5- and Cyanine 3-dUTP nucleotides, respectively, 
using the SureTag DNA Labelling Kit (Agilent Technologies, Santa Clara, CA, USA). Cy5and Cy3-labelled samples were combined in a single mixture and hybridized onto a Human CpG Island Microarray $1 \times 244 \mathrm{~K}$ (Agilent Technologies, Santa Clara, CA, USA) for 40 $\mathrm{h}$ at $67^{\circ} \mathrm{C}$. Microarrays were scanned at $5 \mu \mathrm{m}$ using an Agilent microarray scanner and images analyzed with the Agilent Feature Extraction software v10.7. Data were analyzed by means of the Agilent Genomic Workbench v5.0 software (Agilent Technologies, Santa Clara, CA, USA). The full list of CpG islands (CGIs) analyzed is based on the UCSC Genome Browser hg18, NCBI build 36.1, March 2006. Data were further analyzed according to the methodological approach conceived by Dr. Ravid Straussman and colleagues in 2009 [38]. Briefly, probe z-scores for each CGI were averaged to obtain the Island Methylation Score (IMS) and IMS distribution allowed threshold setting for determining the methylation status of each CGI. CGIs resulted fully methylated (+1) or fully unmethylated $(-1)$ or undetermined (0). Undetermined CGIs were not considered for Gene Ontology analysis.

\subsection{Bionformatic Analysis}

The Gene Ontology analysis was performed using the GOstat software (http:/ / gostat. wehi.edu.au/ accessed on 21 May 2020 [39]) in order to identify the possible enrichment of functional groups related to "biological process", in a specific input list of genes. GOterms were divided in cancer-relevant functional categories as previously described [6]. Analyzed categories were: 'cell cycle', 'cell death and apoptosis', 'response to stimulus', 'cytoskeleton organization', 'cell signalling', 'development and morphogenesis', 'cell differentiation', 'immune response', 'cell motility', 'metabolism', 'transcription and gene expression', 'intracellular transport', 'DNA repair and chromatin remodeling'.

GOstat analysis was also performed narrowing the GO hierarchy through the use of 'biological_function' as a keyword.

Supplementary Materials: The following are available online at https:/ /www.mdpi.com/1422-006 7/22/4/1580/s1, Figure S1: Array-CGH results, Figure S2: Fluorescence in situ hybridization results, Table S1: CNAs shared between STUMPs and LMSs, or STUMPs and LMs, Table S2: Methylation status of gene involved in cell signaling pathway.

Author Contributions: Conceptualization, D.C., S.R., A.A.L., L.D. and A.B.; methodology, D.C., S.R. and A.B.; formal analysis, D.C., S.R. and A.B.; investigation, D.C., S.R., E.G., E.S. and A.B.; resources, A.A.L., P.P., M.P. and F.L.; data curation, D.C., S.R. and A.B.; writing-original draft preparation, D.C., S.R., C.C., L.D. and A.B.; writing-review and editing, D.C., S.R., A.A.L., C.C., P.P., E.G., E.S., M.P., L.D., F.L., M.L., G.R. and A.B.; visualization, D.C., S.R., A.A.L., G.R. and A.B.; supervision, D.C., S.R. and A.B.; project administration, A.B.; funding acquisition, G.R., A.A.L. and A.B. All authors have read and agreed to the published version of the manuscript.

Funding: This research was funded by 2016-ATE-0511 grant from University of Milano-Bicocca (to Gaia Roversi).

Institutional Review Board Statement: The study was conducted according to the guidelines of the Declaration of Helsinki, and approved by the Comitato Etico della provincia Monza e Brianza (protocol code STUMP-1, 26 November 2015).

Informed Consent Statement: Informed consent was obtained from all subjects involved in the study.

Data Availability Statement: Data available on request due to restrictions eg privacy or ethical.

Conflicts of Interest: The authors declare no conflict of interest. 


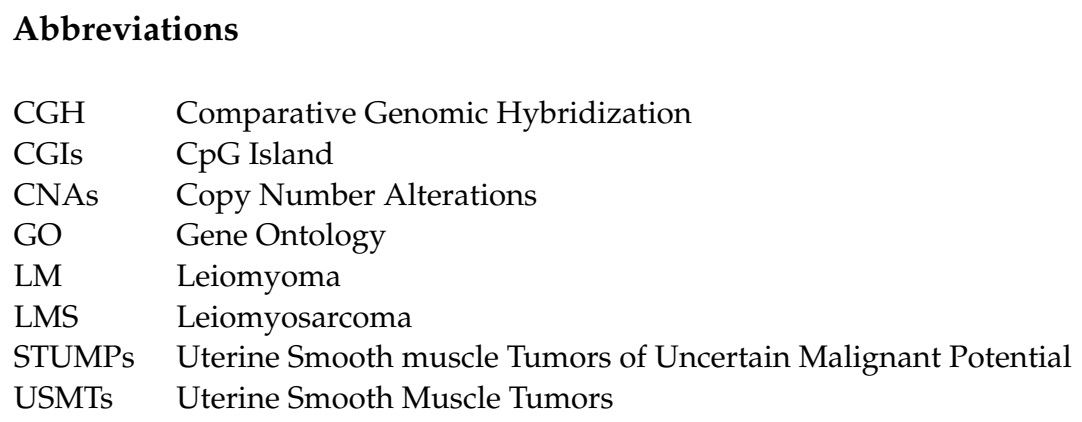

\section{References}

1. Kurman, R.J.; Carcangiu, M.L.; Herrington, C.S.; Young, R.H. WHO Classification of Tumours of Female Reproductive Organs, 4th ed.; WHO Press, World Health Organization: Geneva, Switzerland, 2014; Volume 6.

2. Ip, P.P.; Tse, K.Y.; Tam, K.F. Uterine Smooth Muscle Tumors Other Than the Ordinary Leiomyomas and Leiomyosarcomas: A Review of Selected Variants With Emphasis on Recent Advances and Unusual Morphology That May Cause Concern for Malignancy. Adv. Anat. Pathol. 2010, 17, 91-112. [CrossRef]

3. Gadducci, A.; Zannoni, G.F. Uterine smooth muscle tumors of unknown malignant potential: A challenging question. Gynecol. Oncol. 2019, 154, 631-637. [CrossRef] [PubMed]

4. Croce, S.; Ducoulombier, A.; Ribeiro, A.; Lesluyes, T.; Noel, J.C.; Amant, F.; Guillou, L.; Stoeckle, E.; Devouassoux-Shisheboran, M.; Penel, N.; et al. Genome profiling is an efficient tool to avoid the STUMP classification of uterine smooth muscle lesions: A comprehensive array-genomic hybridization analysis of 77 tumors. Mod. Pathol. 2018, 31, 816-828. [CrossRef]

5. Conconi, D.; Chiappa, V.; Perego, P.; Redaelli, S.; Bovo, G.; Lavitrano, M.; Milani, R.; Dalprà, L.; Lissoni, A.A. Potential role of BCL2 in the recurrence of uterine smooth muscle tumors of uncertain malignant potential. Oncol. Rep. 2017, 37, 41-47. [CrossRef] [PubMed]

6. Baronchelli, S.; Bentivegna, A.; Redaelli, S.; Riva, G.; Butta, V.; Paoletta, L.; Isimbaldi, G.; Miozzo, M.; Tabano, S.; Daga, A.; et al. Delineating the cytogenomic and epigenomic landscapes of glioma stem cell lines. PLoS ONE 2013, 8, e57462. [CrossRef] [PubMed]

7. Ha, H.I.; Choi, M.C.; Heo, J.H.; Kim, K.A.; Jung, S.G.; Park, H.; Joo, W.D.; Song, S.H.; Kim, T.H.; Lee, C. A clinicopathologic review and obstetric outcome of uterine smooth muscle tumor of uncertain malignant potential (STUMP) in a single institution. Eur. J. Obstet. Gynecol. Reprod. Biol. 2018, 228, 1-5. [CrossRef]

8. Cornell, L.; Munck, J.M.; Alsinet, C.; Villanueva, A.; Ogle, L.; Willoughby, C.E.; Televantou, D.; Thomas, H.D.; Jackson, J.; Burt, A.D.; et al. DNA-PK-A candidate driver of hepatocarcinogenesis and tissue biomarker that predicts response to treatment and survival. Clin. Cancer Res. 2015, 21, 925-933. [CrossRef] [PubMed]

9. Sun, S.; Cheng, S.; Zhu, Y.; Zhang, P.; Liu, N.; Xu, T.; Sun, C.; Lv, Y. Identification of PRKDC (Protein Kinase, DNA-Activated, Catalytic Polypeptide) as an essential gene for colorectal cancer (CRCs) cells. Gene 2016, 584, 90-96. [CrossRef] [PubMed]

10. Zhang, Y.; Wen, G.M.; Wu, C.A.; Jing, Z.L.; Li, D.Z.; Liu, G.L.; Wei, X.X.; Tang, M.S.; Li, Y.H.; Zhong, Y.; et al. PRKDC is a prognostic marker for poor survival in gastric cancer patients and regulates DNA damage response. Pathol. Res. Pract. 2019, 215, 152509. [CrossRef]

11. Zhang, Y.; Yang, W.K.; Wen, G.M.; Tang, H.; Wu, C.A.; Wu, Y.X.; Jing, Z.L.; Tang, M.S.; Liu, G.L.; Li, D.Z.; et al. High expression of PRKDC promotes breast cancer cell growth via p38 MAPK signaling and is associated with poor survival. Mol. Genet. Genom. Med. 2019, 7, e908. [CrossRef] [PubMed]

12. Wang, L.; Shilatifard, A. UTX Mutations in Human Cancer. Cancer Cell 2019, 35, 168-176. [CrossRef]

13. Shevchenko, V.; Arnotskaya, N.; Zaitsev, S.; Sharma, A.; Sharma, H.S.; Bryukhovetskiy, A.; Pak, O.; Khotimchenko, Y.; Bryukhovetskiy, I. Proteins of Wnt signaling pathway in cancer stem cells of human glioblastoma. Int. Rev. Neurobiol. 2020, 151, 185-200. [PubMed]

14. Li, M.; Ke, J.; Wang, Q.; Qian, H.; Yang, L.; Zhang, X.; Xiao, J.; Ding, H.; Shan, X.; Liu, Q.; et al. Upregulation of ROCK2 in gastric cancer cell promotes tumor cell proliferation, metastasis and invasion. Clin. Exp. Med. 2017, 17, 519-529. [CrossRef]

15. Hu, J.; Wang, L.; Zhao, W.; Huang, Y.; Wang, Z.; Shen, H. mi-R4435-2HG promotes proliferation and inhibits apoptosis of cancer cells in ovarian carcinoma by upregulating ROCK2. Oncol. Lett. 2020, 19, 1305-1309. [CrossRef] [PubMed]

16. Deng, X.; Yi, X.; Deng, J.; Zou, Y.; Wang, S.; Shan, W.; Liu, P.; Zhang, Z.; Chen, L.; Hao, L. ROCK2 promotes osteosarcoma growth and metastasis by modifying PFKFB3 ubiquitination and degradation. Exp. Cell Res. 2019, 385, 111689. [CrossRef] [PubMed]

17. Mijanović, O.; Branković, A.; Panin, A.N.; Savchuk, S.; Timashev, P.; Ulasov, I.; Lesniak, M.S. Cathepsin B: A sellsword of cancer progression. Cancer Lett. 2019, 449, 207-214. [CrossRef]

18. Ho, K.H.; Cheng, C.H.; Chou, C.M.; Chen, P.H.; Liu, A.J.; Lin, C.W.; Shih, C.M.; Chen, K.C. miR-140 targeting CTSB signaling suppresses the mesenchymal transition and enhances temozolomide cytotoxicity in glioblastoma multiforme. Pharm. Res. 2019, 147, 104390. [CrossRef] [PubMed]

19. Verhoeven, Y.; Tilborghs, S.; Jacobs, J.; De Waele, J.; Quatannens, D.; Deben, C.; Prenen, H.; Pauwels, P.; Trinh, X.B.; Wouters, A.; et al. The potential and controversy of targeting STAT family members in cancer. Semin. Cancer Biol. 2020, 6, 41-56. [CrossRef] 
20. Yang, M.; Chen, H.; Zhou, L.; Chen, K.; Su, F. Expression profile and prognostic values of STAT family members in non-small cell lung cancer. Am. J. Transl. Res. 2019, 11, 4866-4880.

21. Wang, Y.; Sun, W.; Yang, J.; Yang, L.; Li, C.; Liu, H.; Liu, X.; Jiao, B. PUM2 Promotes Glioblastoma Cell Proliferation and Migration via Repressing BTG1 Expression. Cell Struct. Funct. 2019, 44, 29-39. [CrossRef]

22. Zhang, L.; Chen, Y.; Li, C.; Liu, J.; Ren, H.; Li, L.; Zheng, X.; Wang, H.; Han, Z. RNA binding protein PUM2 promotes the stemness of breast cancer cells via competitively binding to neuropilin-1 (NRP-1) mRNA with miR-376a. Biomed. Pharm. 2019, 114, 108772. [CrossRef]

23. Giannini, G.; Ambrosini, M.I.; Di Marcotullio, L.; Cerignoli, F.; Zani, M.; MacKay, A.R.; Screpanti, I.; Frati, L.; Gulino, A. EGF- and cell-cycle-regulated STAG1/PMEPA1/ERG1.2 belongs to a conserved gene family and is overexpressed and amplified in breast and ovarian cancer. Mol. Carcinog. 2003, 38, 188-200. [CrossRef]

24. Xiang, J.; Fang, L.; Luo, Y.; Yang, Z.; Liao, Y.; Cui, J.; Huang, M.; Yang, Z.; Huang, Y.; Fan, X.; et al. Levels of human replication factor C4, a clamp loader, correlate with tumor progression and predict the prognosis for colorectal cancer. J. Transl. Med. 2014, 12, 320. [CrossRef] [PubMed]

25. Yang, W.X.; Pan, Y.Y.; You, C.G. CDK1, CCNB1, CDC20, BUB1, MAD2L1, MCM3, BUB1B, MCM2, and RFC4 May Be Potential Therapeutic Targets for Hepatocellular Carcinoma Using Integrated Bioinformatic Analysis. Biomed. Res. Int. 2019, 2019 , 1245072. [CrossRef] [PubMed]

26. Chung, I.H.; Liu, H.; Lin, Y.H.; Chi, H.C.; Huang, Y.H.; Yang, C.C.; Yeh, C.T.; Tan, B.C.; Lin, K.H. ChIP-on-chip analysis of thyroid hormone-regulated genes and their physiological significance. Oncotarget 2016, 7, 22448-22459. [CrossRef]

27. Liu, Y.; Tao, Z.; Qu, J.; Zhou, X.; Zhang, C. Long non-coding RNA PCAT7 regulates ELF2 signaling through inhibition of miR-134-5p in nasopharyngeal carcinoma. Biochem. Biophys. Res. Commun. 2017, 491, 374-381. [CrossRef] [PubMed]

28. Esteller, M. Aberrant DNA methylation as a cancer-inducing mechanism. Annu. Rev. Pharm. Toxicol. 2005, 45, 629-656. [CrossRef] [PubMed]

29. Feinberg, A.P.; Vogelstein, B. Hypomethylation distinguishes genes of some human cancers from their normal counterparts. Nature 1983, 301, 89-92. [CrossRef] [PubMed]

30. Gaudet, F.; Hodgson, J.G.; Eden, A.; Jackson-Grusby, L.; Dausman, J.; Gray, J.W.; Leonhardt, H.; Jaenisch, R. Induction of tumors in mice by genomic hypomethylation. Science 2003, 300, 489-492. [CrossRef]

31. Hoffmann, M.J.; Schulz, W.A. Causes and consequences of DNA hypomethylation in human cancer. Biochem. Cell Biol. 2005, 83, 296-321. [CrossRef]

32. Cui, H.; Onyango, P.; Brandenburg, S.; Wu, Y.; Hsieh, C.L.; Feinberg, A.P. Loss of imprinting in colorectal cancer linked to hypomethylation of H19 and IGF2. Cancer Res. 2002, 62, 6442-6446.

33. Laner, T.; Schulz, W.A.; Engers, R.; Müller, M.; Florl, A.R. Hypomethylation of the XIST gene promoter in prostate cancer. Oncol. Res. 2005, 15, 257-264. [CrossRef] [PubMed]

34. Bell, S.W.; Kempson, R.L.; Hendrickson, M.R. Problematic uterine smooth muscle neoplasms. A clinicopathologic study of 213 cases. Am. J. Surg. Pathol. 1994, 18, 535-558. [CrossRef] [PubMed]

35. Gupta, M.; Laury, A.L.; Nucci, M.R.; Quade, B.J. Predictors of adverse outcome in uterine smooth muscle tumours of uncertain malignant potential (STUMP): A clinicopathological analysis of 22 cases with a proposal for the inclusion of additional histological parameters. Histopathology 2018, 73, 284-298. [CrossRef]

36. Ip, P.P.; Cheung, A.N.; Clement, P.B. Uterine smooth muscle tumors of uncertain malignant potential (STUMP): A clinicopathologic analysis of 16 cases. Am. J. Surg. Pathol. 2009, 33, 992-1005. [CrossRef] [PubMed]

37. Cheung, S.W.; Shaw, C.A.; Scott, D.A. Microarray-based CGH detects chromosomal mosaicism not revealed by conventional cytogenetics. Am. J. Med. Genet. A 2007, 143, 1679-1686. [CrossRef]

38. Straussman, R.; Nejman, D.; Roberts, D.; Steinfeld, I.; Blum, B.; Benvenisty, N.; Simon, I.; Yakhini, Z.; Cedar, H. Developmental programming of CpG island methylation profiles in the human genome. Nat. Struct. Mol. Biol. 2009, 16, 564-571. [CrossRef]

39. Beissbarth, T.; Speed, T.P. GOstat: Find statistically overrepresented Gene Ontologies within a group of genes. Bioinformatics 2004, 20, 1464-1465. [CrossRef] 